\title{
Evaluation of Wheat Genotypes for a Single Stem Rust Race TTTTF in Ethiopia
}

\author{
Lidiya Tilahun Hadis ${ }^{1,}$, Tamirat Negash Gure ${ }^{1}$, Daniel Kassa Habtemariam ${ }^{1}$, Getnet Muche Abebile ${ }^{1}$, \\ Fikrte Yirga Belayineh $^{2}$, Alemu Ayele Zerihun ${ }^{1}$ \\ ${ }^{1}$ Ethiopian Institute of Agricultural Research (EIAR), Kulumsa Agricultural Research Center, Assela, Ethiopia \\ ${ }^{2}$ Ethiopian Institute of Agricultural Research (EIAR), Addis Ababa, Ethiopia
}

\section{Email address:}

lidtilahun2015@gmail.com (L. T. Hadis), tamnegu@gmail.com (T. N. Gure), danieyobe@yahoo.com (D. K. Habtemariam), getnetmuche2014 (G. M. Abebile), fikryirga19@gmail.com (F. Y. Belayineh), alemuayele81@gmail.com (A. A. Zerihun)

${ }^{*}$ Corresponding author

\section{To cite this article:}

Lidiya Tilahun Hadis, Tamirat Negash Gure, Daniel Kassa Habtemariam, Getnet Muche Abebile, Fikrte Yirga Belayineh, Alemu Ayele Zerihun. Evaluation of Wheat Genotypes for a Single Stem Rust Race TTTTF in Ethiopia. Biomedical Statistics and Informatics.

Vol. 6, No. 3, 2021, pp. 47-53. doi: 10.11648/j.bsi.20210603.12

Received: July 21, 2021; Accepted: August 4, 2021; Published: August 23, 2021

\begin{abstract}
Stem rust caused by Puccinia graminis is one of the most destructive diseases of wheat which causing considerable yield losses in wheat-growing areas in Ethiopia. Disease-resistant cultivars provide one of the best means for controlling stem rust. The current study aimed to evaluate wheat genotypes for seedling and adult plant stage resistance for the predominant wheat stem rust races; TTTTF. Both seedling and adult plant stage evaluations were conducted on 203 wheat genotypes under greenhouse and field conditions. Seedling and adult plant stage evaluations were conducted using artificial inoculation of the race TTTTF. Augmented and completely randomized designs were used for field and seedling tests, respectively. Three susceptible and two resistance check genotypes were used for field evaluation for each block. Seedling evaluation results showed $85 \%$ of the genotypes exhibited resistance infection type $(<2)$. Field phenotyping results revealed $72 \%, 22 \%$ and $6 \%$ of the genotypes showed resistance $(<15 \%)$, moderately resistance $(20-35 \%)$ and susceptible $(>40)$ reaction for stem rust race TTTTF. From the ANOVA table, highly significant variation between test and check genotypes was observed for different disease parameters and TKW. High and positive correlations existed between different disease parameters and a negative correlation was observed between TKW and disease parameters. Based on field and seedling evaluation, a total of 151 genotypes were selected for future breeding pipelines. Therefore, resistant wheat genotypes identified in the current study will be a potential source of genotypes for further wheat breeding and pathological research activities.
\end{abstract}

Keywords: Wheat, Stem Rust, Race, Resistance

\section{Introduction}

Wheat is one of the most important food security crops in the world, accounting for a sizeable share of the global calories and protein consumed [1]. Similarly, it is a staple food in the diets of several Ethiopian, providing about $15 \%$ of the caloric intake for the country's over 90 million populations [2], placing it second after Maize and slightly ahead of Teff, Sorghum, and Enset, which contribute 10 to 12 percent each [3]. Bread wheat (Triticum aestivum L. Thell) and durum wheat (Triticum. turgidum L. var. durum) are the two main wheat species cultivated in Ethiopia [4].
According to [5] report, the land cultivated to wheat is increased to 1.69 million hectares and production has boosted to 46.42 million quintals with a productivity of $2.73 \mathrm{t} \mathrm{ha}^{-1}$, which is still below the world's average of $3.3 \mathrm{t} \mathrm{ha}^{-1}$ [2]. About 4.21 million smallholder farmers (SHFs) engaged in wheat production in the country and most of the wheat production comes from the SHFs. The central and southeastern highlands (Arsi and Bale zones) of the country are considered as a wheat belt area of the country. Despite the enormous economic and dietary values of the crop, the production of wheat in Ethiopia remained low as compared to the demand and still, wheat importing is the main source of wheat self-sufficiency in the country. Biotic and abiotic factors are the main source of 
bottlenecks in Ethiopia for wheat production [4]. Rust diseases (stem, stripe, and leaf rust) are the major and economically important biotic factors for wheat production in Ethiopia [6, 7].

Rusts mainly stem (Puccinia graminisf. sp. tritici) and yellow rusts, have been considered the most important wheat diseases in Ethiopia, and thus, have been one of the most important priority research agenda since the inception of wheat research on wheat diseases [6]. Stem rust or black rust caused by Puccinia graminisf. sp. tritici is amongst the rust diseases that can cause up to $100 \%$ yield loss if susceptible cultivars are grown and epidemic occurs $[6,8]$. Stem rust isolates with virulence to $\mathrm{Sr} 9 \mathrm{e}$ and $\mathrm{Sr} 13$ were first reported in Ethiopia in 1988 and 1989, respectively [6]. The highlands of Ethiopia are considered as a hot spot for the development of stem rust races and so far more than nine races were identified, some of the races are TTKSK, TTKTF, TTKTK, JRCQC, TKTTF, TTKSC, TRTTF, SRKSC and RRKSF [9].

Stem rust is known to occur all over the world, in areas where wheat is produced but the damage and economic impact depends on the environmental conditions and the types of cultivars grown. Host resistance and chemical option are the two major rust management practices in Ethiopia and worldwide. Host resistance (genetic control) has advantages for environmental and economic reasons, particularly for farmers in the developing world, and because of the possibility that rust pathogens develop resistance to fungicides [10]. Resistance conditioned by major genes has been the most widely emphasized strategy for mitigating rust threats and reducing losses incurred. Pyramiding of several major genes into a single cultivar is also an attractive breeding strategy for increasing resistance durability by reducing the stepwise accumulation of virulence by the pathogen against each gene. The alternative is the development and employment of cultivars carrying durable or slow rusting resistance based on quantitatively inherited, multiple genes referred to as adult plant resistance $[10,11]$.
In Ethiopia, the wheat improvement program started before half a century [4], and more than 100's of wheat varieties was released for production [5]. However, currently, very few of the varieties are under production, and others are beaten by different races of stem and yellow rusts in different periods [4, 12-16]. Developing durable rust-resistance wheat genotypes demands both seedling and adult plant stage evaluation using major wheat rust races. In this case, it necessitates, new strategy apart from the traditional way of rust resistance evaluation for adult plant stage using single stem rust screening nurseries. Therefore, the objective of this study is to evaluate wheat genotypes at seedling and adult plant stage for wheat stem rust race "TTTTF" in Ethiopia.

\section{Materials and Methods}

\subsection{Description of Experimental Sites and Genotypes}

Field phenotyping for single stem rust nursery was conducted at Kulumsa Agriculture Research Center (KARC) which is located at $08^{\circ} 02^{\prime} \mathrm{N}$ latitude and $39^{\circ} 09$ E longitude and at an altitude of 2250 m.a.s.l. The monthly mean minimum and maximum temperatures are $10.5^{\circ} \mathrm{C}$ and $22.8^{\circ} \mathrm{C}$, respectively and the average rainfall is $840 \mathrm{~mm}$. Seedling stage evaluation for a single stem rust race under control environment was conducted at Ambo Agricultural Research Center. It is located at $08^{\circ} 96^{\prime} 885^{\prime \prime} \mathrm{N}$ latitude and 370 85' $923^{\prime \prime}$ E longitude and at an altitude of 2147 m.a.s.l. The annual average temperature and rainfall are $27.54^{\circ} \mathrm{C}$ and $1077.68 \mathrm{~mm}$ respectively.

A total of 203 (168 bread wheat and 35 durum wheat) genotypes were included in the study. The genotypes are composed from known susceptible and resistance checks for stem rust, deferential lines, released varieties and breeding genotypes. The details of the study genotypes described in table 1.

Table 1. Description of the study wheat genotypes.

\begin{tabular}{|c|c|c|c|c|c|}
\hline \multirow{2}{*}{ Species } & \multicolumn{2}{|c|}{ \# of test lines } & \multirow{2}{*}{ \# of Checks } & \multirow{2}{*}{ \#of Differentials } & \multirow{2}{*}{ Total } \\
\hline & Variety & Elite line & & & \\
\hline Bread Wheat & 31 & 121 & 4 & 12 & 168 \\
\hline Durum Wheat & 3 & 31 & 1 & - & 35 \\
\hline Total & 34 & 152 & 5 & 12 & 203 \\
\hline
\end{tabular}

\subsection{Experimental Methodology}

Field phenotyping for single stem rust nursery: In Ethiopia, several virulence stem rust races were emerged in different periods. Stem rust races "TTTTF" is one of the dominant races in different wheat growing areas of Ethiopia [17, 18]. It is virulence for stem rust genes $5,21,9 e, 7 b, 11,6,8 a, 9 g, 36,9 b$ $30,17,9 a, 9 d, 10, T m p, 38, M c N$ and avirulence only for 24 and 31. Therefore, a single stem rust nursery (race TTTTF) was established to evaluate a set of 203 genotypes (Table 1) in the 2018 main cropping season. The experiment was laid out in augmented design with eight blocks with five susceptible, moderate, and resistant checks replicated in each block. Two rows of stem rust susceptible lines "Digalu" used as infector row and planted perpendicular (between blocks) two weeks before entries were planted. The distance between spreader rows and spacing between spreader rows and entries was kept at $0.5 \mathrm{~m}$ and $0.2 \mathrm{~m}$, respectively. Borders of tall Oat variety and Yellow dodder were drilled around the nursery to serve as an experimental guard rows border against incoming rust spores. Testing entries were planted in double rows of 0.5 -meter-long and $0.2 \mathrm{~m}$ spacing between rows. Weeding and other agronomic practices were applied according to recommended practices for wheat production of Kulumsa. The spreader rows were inoculated with spores of stem rust race TTTTF six times starting from stem elongation using injection and spraying 
methods [19]. One injection and five times spray were made in different growth stage of the wheat plant. Disease severity and infection response were visually assessed four times starting from the onset of disease on the entries using modified Cobb scale [20].

Seedling stage evaluation: The same genotypes were evaluated for seedling stage against stem rust race TTTTF at Ambo Agricultural Research Center (AARC). Five seeds of each genotype were planted in a pot $(4 \mathrm{~cm}$ diameter $)$ separately, using CRD (Completely Randomized Design) in two replications. Seven-days-old seedlings were inoculated with spores of stem rust race TTTTF. For incubation, inoculated plants were moistened with fine droplets of distilled water by using an atomizer. After 20 minutes of inoculation, the seedlings were placed in dew chamber for 18 hrs in a dark at $18-22^{\circ} \mathrm{C}$. Upon removal from the chamber, seedlings were exposed to $3 \mathrm{hrs}$ of fluorescent light to dry dew on the leaves. Following this, the seedlings were transferred to the greenhouse where conditions were regulated at $12 \mathrm{hrs}$ photoperiod, at temperature range of $18-25^{\circ} \mathrm{C}$ and $\mathrm{RH}$ of $60-70 \%$ [21]. Susceptible check variety called "McNair" was used to confirm the success of inoculation. Data on infection types (ITs) were recorded 14 days after inoculation using 0-4 scale based on [22].

\subsection{Data Analysis}

Final Rust Severity (FRS), Final Coefficient of Infection (FCI), Average Coefficient of Infection (ACI) and Area Under Disease Progress Curve (AUDPC) were used from field phenotyping data and average seedling infection type from the seedling test as criterion to identify any possible source of partial resistance to stem rust race TTTTF. Analysis of variance (ANOVA) was conducted to determine differences among the wheat genotypes for different disease parameters. Simple descriptive statistics and Pearson correlation coefficient was used to assess the relationship between different disease parameters and yield associated trait called thousand kernel weight (TKW). All statistical analyses and graphs were performed using excel 2013 and R version 4.0.2 [23].

\section{Results and Discussions}

The field phenotyping and seedling test result revealed phenotypic variability for severity and infection types between test genotypes and checks. The final severity score ranged from 0 (Immune/resistance) to 40 (Susceptible). The response of 198 bread and durum wheat genotypes and the five checks for the single stem rust race TTTTF field and seedling stage evaluation summarized in Figure 1. From adult stage phenotyping, $72 \%, 22 \%$, and $6 \%$ of genotypes showed moderate resistance $(<15 \%$ severity), moderately susceptible (20-35\% severity), and susceptible reaction ( $>40 \%$ severity) for stem rust race TTTTF, respectively. The checks showed variable reaction responses from moderately resistant to susceptible and a severity level ranging from 10 to $90 \%$. From the seedling test, $85 \%$ of the genotype showed resistant reaction (infection type $<2$ ) and $15 \%$ of the genotypes scored $>2$ infection type and considered as a seedling susceptible for stem rust race TTTTF.

The frequency of test and check genotypes for different disease measurement parameters and TKW showed in figure 2 From check genotypes, the highest mean value for FRS, FCI, ACI and AUDPC scored from Arendeto and Digalu. This indicates these two check genotypes were highly susceptible for stem rust race TTTTF. While Kingbird and Danda'a scored the lowest mean value for all disease parameters and showed resistant reaction for stem rust race TTTTF. Due to low susceptibility reaction, Danda'a and Kingbird scored the highest TKW among check genotypes. The highest TKW 46.5, 45.8 and 45.7 scored from genotype ETBW9463, ETBW9292 and ETBW9581, respectively. These genotypes were showed highly resistance reaction for race TTTTF under field condition. The lowest TKW scored from Digalu, Arendeto and KBG-01 with 10.0, 11.3 and 11.8, respectively.

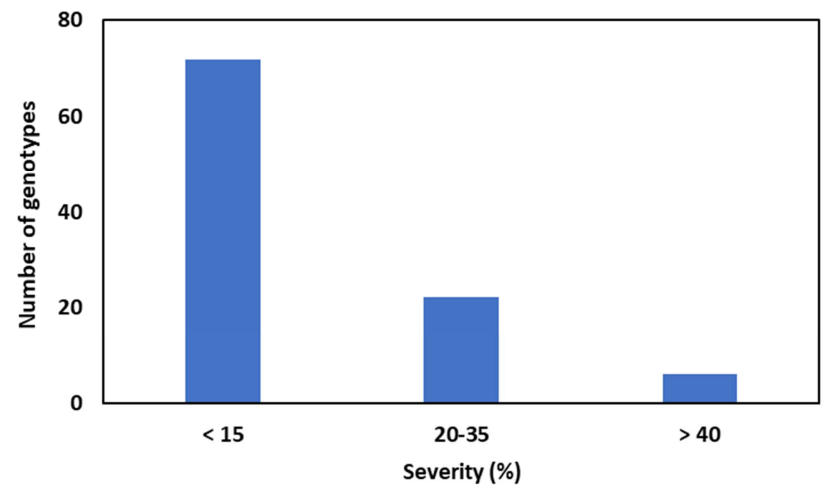

(A)

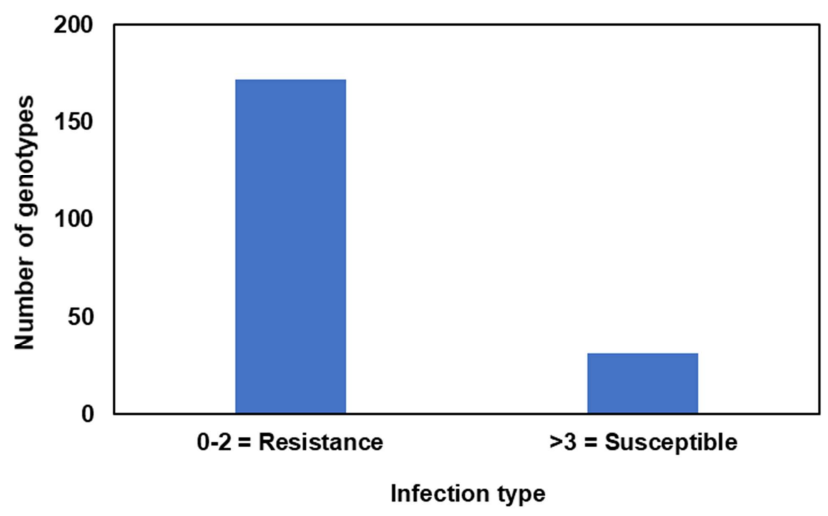

(B)

Figure 1. Severity response of wheat genotypes for single stem rust race TTTTF under adult plant (A) and seedling stage evaluation (B).

Different disease measurement parameters derived from the final disease severity and reaction response showed significant phenotypic variation between the test genotypes and the checks used in this study for stem rust race TTTTF (Table 2). This indicates the variability within the test and check genotypes for race TTTTF virulence. In addition, TKW showed highly significant variation $(\mathrm{P}<0.01)$ between test 
and checks genotypes. The minimum and maximum value of TKW were 10.5 to 46.5 gram per thousand seed weight, respectively. Adjusted mean value for test and check genotypes for ACI, AUDPC, FCI, FRS and TKW were 4.71,
$211.48,9.52,12.11$ and 32.11 , respectively. Based on the field phenotyping and seedling test result, 151 genotypes were selected based on their final severity $<20 \%$ and seedling infection $<2$.
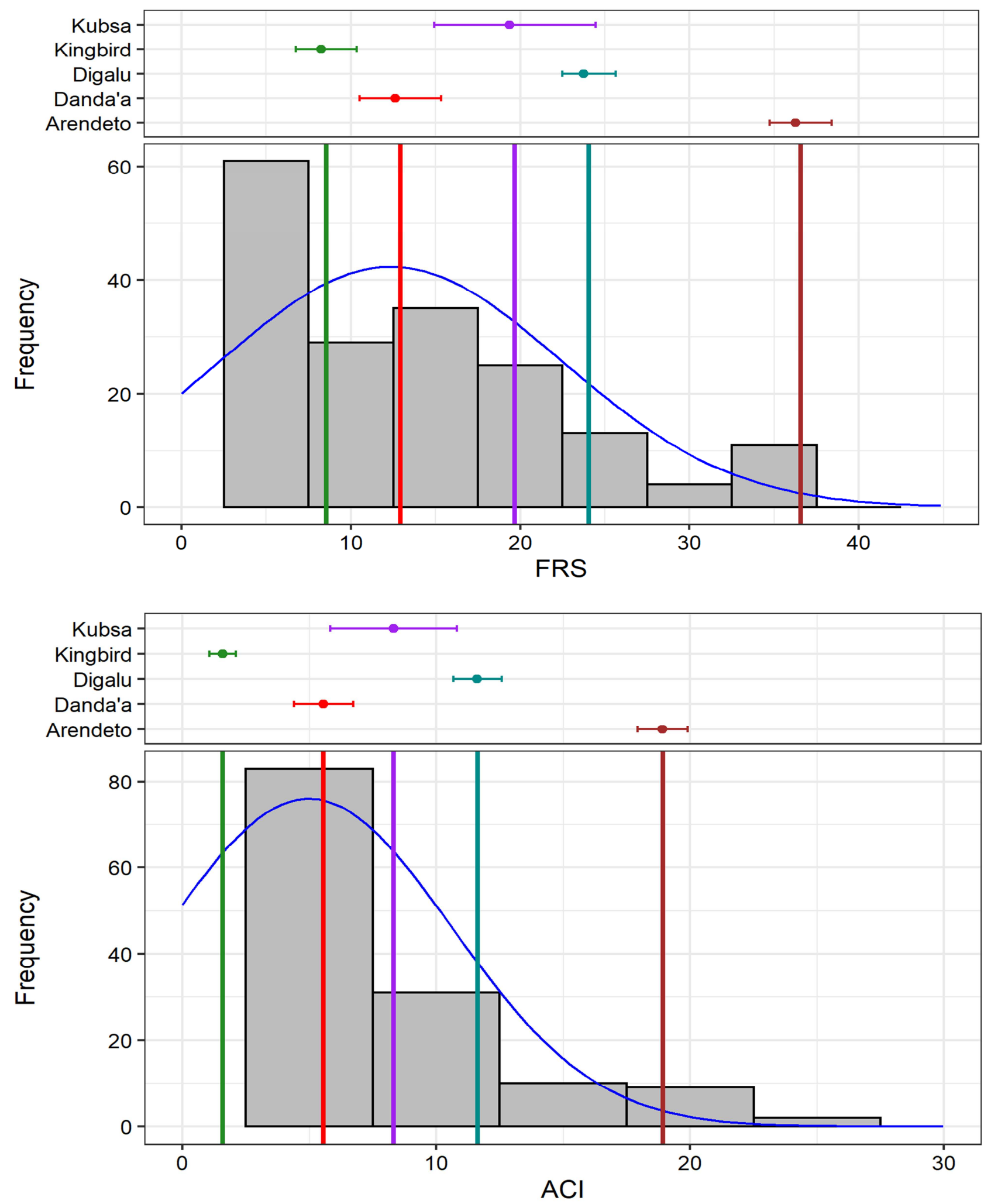

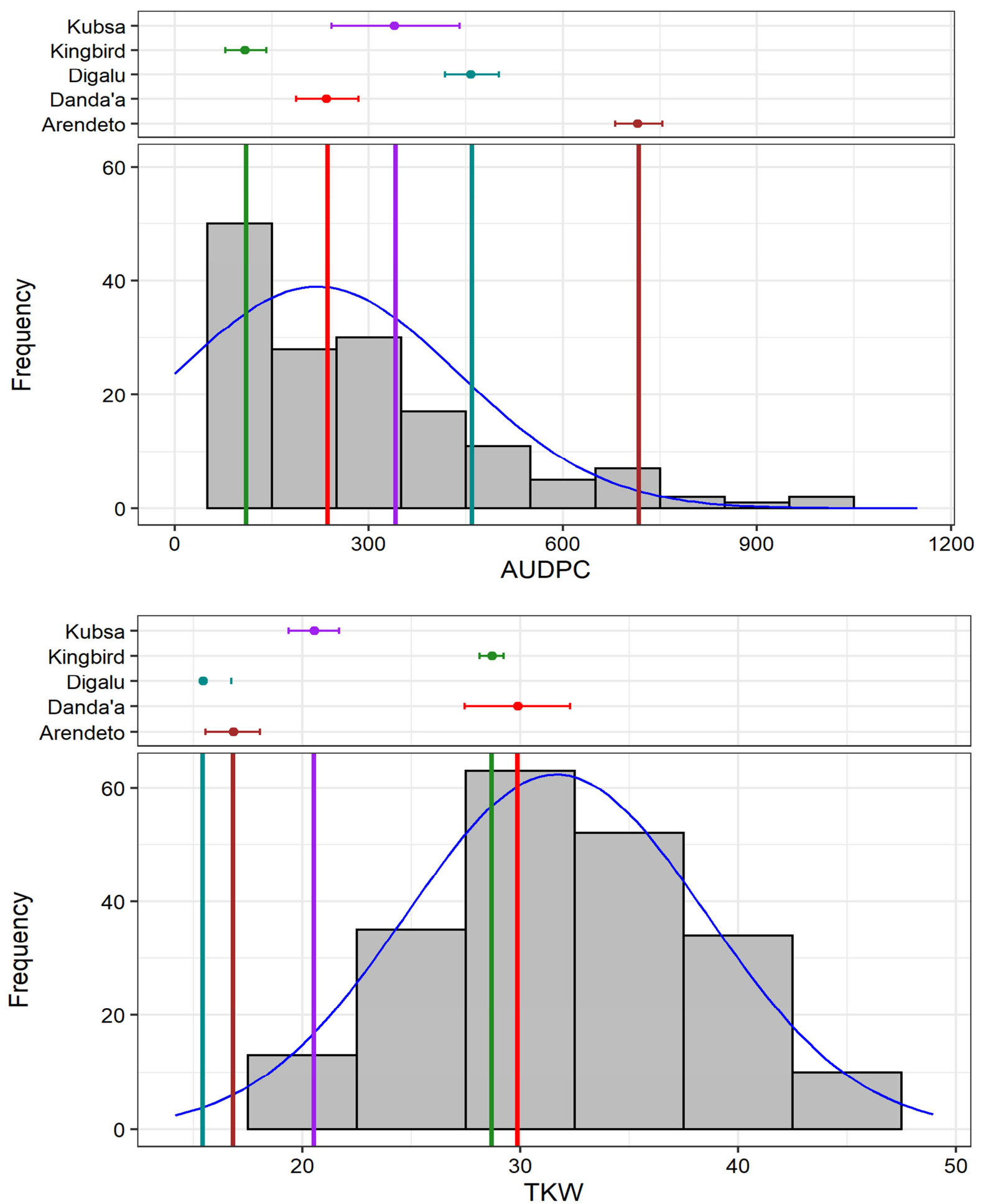

Figure 2. Frequency of test and checks genotypes for different disease parameters and TKW. Check genotypes response showed in the upper part of each graphs.

Pearson correlation matrix between different disease parameters, seedling test and TKW showed in figure 3 . Positive and significant correlation (0.9) observed between ACI, AUDPC, FCI and FRS. This indicates similar response of the genotypes for different disease parameters. The seedling test showed positive and non-significant correlation between ACI and AUDPC. Negative and non-significant correlation observed between TKW and seedling test. TKW is negatively correlated with all disease parameters (Figure 3). Similar results were reported by Hundie et al [24] using four predominant stem rust races; TTKSK, TRTTF, TKTTF and JRCQC. 
Table 2. ANOVA table highlighting levels of significance observed among various disease parameters and TKW for test and check genotypes.

\begin{tabular}{|c|c|c|c|c|c|c|}
\hline Source & Df & ACI & AUDPC & FCI & FRS & TKW \\
\hline Treatment (ignoring Blocks) & 202 & $46.61 * *$ & $68101.85 * *$ & $156.29 * *$ & $145.98 * *$ & $68.35 * *$ \\
\hline Treatment: Check & 4 & $344.45 * *$ & $429668.98 * *$ & $1203.06 * *$ & $941.91 * *$ & $357.59 * *$ \\
\hline Treatment: Test vs. Check & 1 & $711.41 * *$ & $894877.04 * *$ & $2877.7 * *$ & $2219.47 * *$ & $3391.38 * *$ \\
\hline Block (eliminating Treatments) & 4 & $89.95 * *$ & $157671.14 * *$ & $316.41 * *$ & $381.59 * *$ & $58.45 * *$ \\
\hline Residuals & 43 & 14.75 & 23304.9 & 49.7 & 45.28 & 11.14 \\
\hline $\mathrm{CV}$ & & 71.9 & 65.09 & 65.48 & 50.84 & 11.00 \\
\hline
\end{tabular}

ns $\mathrm{P}>0.05 ; * \mathrm{P}<0.05 ; * * \mathrm{P}<0.01$

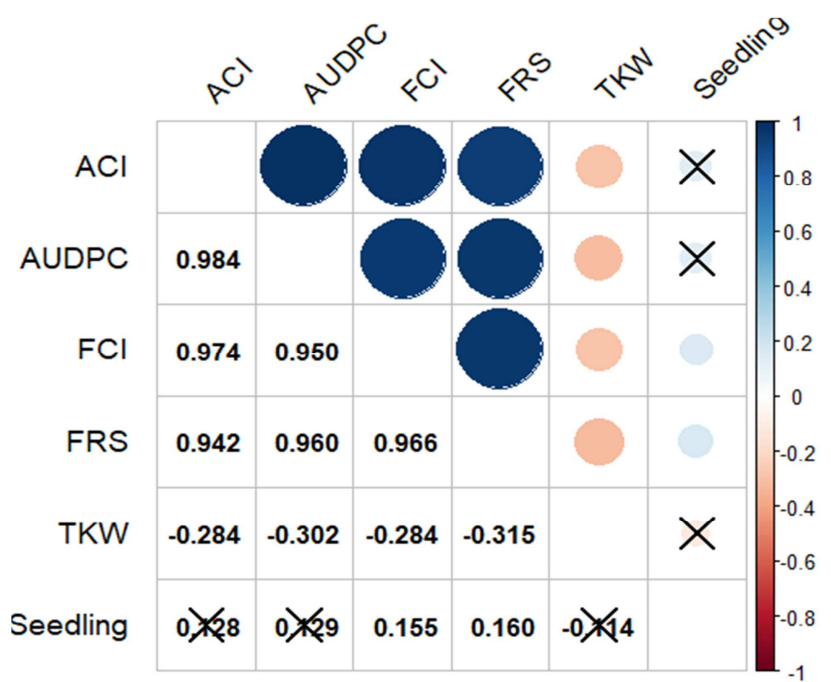

Figure 3. Pearson correlation matrix for different adult plant disease parameters, seedling test and TKW.

\section{Conclusion}

In this study, a total of 203 wheat genotypes (test, checks and deferential lines) were successfully evaluated at seedling and adult plant stage for a single stem rust race TTTTF. High phenotypic variation was observed for both seedling and adult plant stage evaluation between test, check and deferential lines used in this study. Positive and significant correlation observed between different disease parameters. But the correlation between disease parameters and TKW showed negative correlation. Negative and non-significant correlation observed between seedling stage evaluation and TKW.

The result clearly indicates the importance of wheat genotypes evaluation at seedling and adult plant stages for a single predominant stem rust race to identify. A total of 151 genotypes were selected based on their field and seedling evaluation and these genotypes will be a source of future wheat improvement pipeline for breeding and pathological research activities.

\section{Acknowledgements}

The authors acknowledge Delivering Genetic Gain in Wheat (DGGW) project for financial support of this study. A special gratitude to Dr. Matthew Rouse (USDA-ARS), who introduces and trained wheat researchers about single stem rust screening nursery establishment principles at Kulumsa
Agricultural Research center. The Ethiopian Institute of Agricultural Research (EIAR) is acknowledged for hosting the field research of this study.

\section{References}

[1] Shiferaw, B., et al., Crops that feed the world 10. Past successes and future challenges to the role played by wheat in global food security. Food Security, 2013. 5 (3): p. 291-317.

[2] FAOSTAT, Wheat production and area harvested. 2017.

[3] Minot, N., et al., The wheat supply chain in Ethiopia: Patterns, trends, and policy options. F1000Research, 2015.3.

[4] Hailu, G., Wheat Production and Research in Ethiopia. 1991.

[5] CSA, Agricultural Sample Survey. Report on area and production of major crops. 2017/18, Central Statistical Authority (CSA). Addis Ababa, Ethiopia. p. 14.

[6] Mengistu, H., et al., Wheat pathology research in Ethiopia. 1991.

[7] Ayele, B., et al., Review of two decades of research on diseases of small cereal crops. 2008.

[8] Ayele, B. H., Breeding bread wheat with multiple disease resistance and high yield for the Ethiopian highlands: broadening the genetic basis of yellow rust and tan spot resistance. 2002, Gottingen University, Germany: Germany.

[9] Hailu, E., et al., Distribution of Stem Rust (Puccinia graminis f. sp. tritici) Races in Ethiopia. Advances in Crop Science and Technology, 2015: p. 1-4.

[10] Olivera, P., et al., Phenotypic and Genotypic Characterization of Race TKTTF of Puccinia graminis f. sp. tritici that Caused a Wheat Stem Rust Epidemic in Southern Ethiopia in 2013-14. 2015. 105.

[11] Hei, N., et al., Assessment of Ethiopian Wheat Lines for Slow Rusting Resistance to Stem Rust of Wheat Caused by Puccinia graminisf. sp. tritici. Journal of Phytopathology, 2015. 163 (5): p. 353-363.

[12] Bekele, E. A review of research on diseases of barley, tef and wheat in Ethiopia. in Proceedings of the First Ethiopian Crop Protection Symposium. 1986. Addis Ababa (Ethiopia): IAR.

[13] Mekuria, M. and E. W. Crawford, Econometric-Analysis of Wheat Production Technology Adoption in the Southeastern Highlands of Ethiopia. American Journal of Agricultural Economics, 1994. 76 (5): p. 1252-1252. 
[14] Bekele, H., et al., Adoption of improved wheat technologies in Adaba and Dodola Woredas of the Bale Highlands, Ethiopia. 2000, CIMMYT.

[15] Badebo, A., Breeding bread wheat with multiple disease resistance and high yield for the Ethiopian highlands: broadening the genetic basis of yellow rust and tan spot resistance. 2002, Univcrsity Gottingen.

[16] Bishaw, Z. and D. Alemu, Farmers' perceptions on improved bread wheat varieties and formal seed supply in Ethiopia. International Journal of Plant Production, 2017. 11 (1): p. 117-130.

[17] Hei, N. B., et al., Distribution and frequency of wheat stem rust races (Puccinia graminis f. sp. tritici) in Ethiopia. Journal of Agricultural and Crop Research, 2018. 6 (5): pp. 88-96.

[18] Yesuf, N. S., et al., Distribution, Epidemics dynamics and physiological races of wheat stem rust (Puccinia graminis f. sp. tritici Eriks and E. Henn) on irrigated wheat in the Awash River basin of Ethiopia. bioRxiv, 2021.

[19] Roelfs, A. P., R. P. Singh, and E. E. Saari, Rust diseases of wheat: concepts and methods of disease management. 1992: Cimmyt.
[20] Peterson, R. F., A. Campbell, and A. Hannah, A diagrammatic scale for estimating rust intensity on leaves and stems of cereals. Canadian journal of research, 1948. 26 (5): p. 496-500.

[21] Woldeab, G., E. Hailu, and N. Bacha, Protocols for Race Analysis of Wheat Stem Rust (Puccinia graminis f. sp. tritici). 2017, EIAR, Ambo, Ethiopia. Available online at www. globalrust. org/race-manual/Ambo.

[22] Stakman, E. C., D. Stewart, and W. Loegering, Identification of physiologic races of Puccinia graminis var. tritici. 1962: USDA Washington.

[23] R Core Team, R: A language and environment for statistical computing. R Foundation for Statistical Computing, 2020.

[24] Hundie, B., Girma, B., Tadesse, Z., Edae, E., Olivera, P., Abera, E. H., Bulbula, W. D., Abeyo, B., Badebo, A., Cisar, G. and Brown-Guedira, G., 2019. Characterization of Ethiopian wheat germplasm for resistance to four Puccinia graminis f. sp. tritici races facilitated by single-race nurseries. Plant disease, 103 (9), pp. 2359-2366. 